\title{
Changes of Magnetic Resonance Imaging on the Brain in Beagle Dogs with Aging
}

\author{
Tomofumi KIMOTSUKI ${ }^{1,2)}$, Tsukasa NAGAOKA ${ }^{3)}$, Mitsuya YASUDA ${ }^{1)}$, Satoshi TAMAHARA ${ }^{2)}$, Naoaki MATSUKI ${ }^{2)}$ \\ and Kenichiro $\mathrm{ONO}^{2) *}$ \\ ${ }^{1)}$ Medicinal Safety Research Laboratories, Sankyo Co., Ltd., 717 Horikoshi, Fukuroi, Shizuoka 437-0065, ${ }^{2)}$ Department of Veterinary \\ Clinical Pathobiology, Graduate School of Agricultural and Life Sciences, The University of Tokyo, 1-1-1 Yayoi, Bunkyo-ku, Tokyo 113- \\ 8657 and ${ }^{3)}$ Laboratory Department of Neurosurgery, Tokyo Medical and Dental University, 1-5-45 Yushima, Bunkyo-ku, Tokyo $113-$ \\ 0034, Japan
}

(Received 8 September 2004/Accepted 1 June 2005)

\begin{abstract}
Age-associated changes of magnetic resonance imaging (MRI) on the brain were evaluated in 19 beagle dogs aged from 8month- to 16-year-old. A significant correlation of the volume of lateral ventricle space was observed in the dogs with age advanced, however, no correlation was found between hippocampus size and the aging. The hypo-intensity areas on T2-weighted MRI were detected in globus pallidus and substantia nigra with a significant correlation of both intensity ratios to lateral ventricle with age advanced. These areas were coincided with the accumulation of iron in the slice of the brain with Perls' staining. In addition, hyperintensity area, suggesting perivascular demyelination with fluid-filled space, was also observed in white matter surrounding the lateral ventricle on T2-weighted MRI. These results suggested that age-associated changes of T2-weighted MRI were developed in the dog brain, especially in globus pallidus, substantia nigra, and white matter surrounding lateral ventricle, like as those reported in the human brain
\end{abstract}

KEY WORDS: age-associated change, brain, canine, hypo- and hyper-intensity, MRI.

J. Vet. Med. Sci. 67(10): 961-967, 2005

Magnetic resonance imaging (MRI) has been used as a noninvasive and useful diagnostic technique for evaluating age related brain disorders, such as Alzheimer's disease, Parkinson's disease, and dementia, indicating severe atrophy of the brain, hippocampus, amygdala, and temporal lobe, gray matter, and dilatation of ventricle space, and cortical sulci, [9, 17, 26, 28, 34]. However, age-associated similar changes of MRI was also observed in the clinically healthy human brain, indicating brain atrophy, dilatation of ventricle space and cortical sulci, hypo-intensity area in globus pallidus and substantia nigra, and hyper-intensity area in white matter $[7,16,20,31]$. In addition, age-associated pathological changes of the brain, including developments of senile plaque, decrease number of neuronal cell, deposit of lipofuscin, and activation of astrocyte, have also been well documented [8, 12, 23, 38]. Therefore, it is necessary for diagnose of age related degenerative diseases to evaluate age-associated changes in the brain. Various age-associated changes were detected in aged dogs with occasionally abnormal behaviors $[4,6,10,27,30]$, like as those reported in human [13]. Although MRI has been applied in veterinary clinics and experimental medicine, several reports were on clinical cases and few reports on age-associated changes in the dog brain [5, 37]. This study deals with ageassociated changes in MRI on the brain, especially lateral ventricle space, hippocampus size, and hypo- and hyperintensity area, in beagle dogs with age advanced.

\footnotetext{
* Correspondence to: Ono, K., Department of Veterinary Clinical Pathobiology, Graduate School of Agricultural and Life Sciences, The University of Tokyo, 1-1-1 Yayoi, Bunkyo-ku, Tokyo 113-8657, Japan.
}

\section{MATERIALS AND METHODS}

Animals: Clinically healthy 19 beagle dogs $(8$ male and 11 female), aged from 8-month- to 16-year-old, were used. All the dogs were bred, reared, and kept in Medicinal Safety Research Laboratories, Sankyo Co., Ltd. under the condition of circumstance temperature at $22 \pm 2^{\circ} \mathrm{C}$. The air extraction was designed to be 10 times/h and the lighting control was $12 \mathrm{~L}$ and 12D. Each dog was offered $220 \mathrm{~g}$ of standard diet once a day in the morning. Tap water was given ad libitum by the automatic watering system.

Anesthesia: For the taking MRI, the dogs were administered with atropine sulfate $(0.05 \mathrm{mg} / \mathrm{kg}$, s.c.) and xylazine (2 $\mathrm{mg} / \mathrm{kg}$, i.m.) for the sedation. After the premedication, ketamine hydrochloride $(10 \mathrm{mg} / \mathrm{kg}$, i.v.) was injected and endotracheal tube was inserted. Anesthesia was maintained with isoflurane using a respirator.

Magnetic resonance imaging (MRI): MRI was carried out on a 4.7 tesla $(\mathrm{T})$ experimental imager/spectrometer system (Unity puls SIS 220/300, Varian, PaloAlto, Ca, U.S.A.) using a $33 \mathrm{~cm}$ horizontal bore magnet (Oxford instrument, England) and a gradient strength of $50 \mathrm{mT} / \mathrm{m}$. T1-weighted spin-echo $(\mathrm{SE})$ images $(\mathrm{TR} / \mathrm{TE})(\mathrm{msec}=500 / 20)$ with 4 signal acquisition and T2-weighted SE images (2000/80) with 2 signal acquisition were also performed. The section thickness was $3 \mathrm{~mm}$ with a $256 \times 256$ matrix and a field of view of $15 \times 15 \mathrm{~cm}^{2}$. Transverse images were taken from forebrain to hindbrain.

Evaluation of MRI: From the results of T1- and T2weight SE images, evaluation of MRI was carried out on the T2-weight SE images. The data of MRI were transferred into the computer and stored. The regions of interest, lateral 
ventricle and hippocampus, were determined by hand-drawing manner for calculating the size of the area by the computer using software (ULTIMAGE, GRAFTEK, Tokyo). Total volume of lateral ventricle space was calculated from the sum total of the size of area timed a thickness of the slice (3.5 mm corrected as the section thickness and gap were 3 $\mathrm{mm}$ and $0.5 \mathrm{~mm}$, respectively) in every sections. Hippocampus size was also measured in the section, in which it was shown in the largest view. For hypo-intensity area in the section at globus pallidus and substantia nigra, and hyperintensity area in white matter on T2-weighted images were evaluated the signal intensity of those area compared to that of lateral ventricle.

Pathological examination: After the taking MRI, one male beagle dog, aged 16-year-old, was killed under general anesthesia with sodium pentobarbital and autopsied. The postmortem brain was fixed with $10 \%$ neutral buffered formalin. The fixed brain was sliced in the transverse plane at the level of globus pallidus and substantia nigra, and stained with Perls' solution to detect the ferric iron, because hypointensity area on T2-weighted MRI was demonstrated to be closely related to the iron accumulation [14].

Statistic analysis: Quadratic regression analysis was used for statistical significance between the aging and lateral ventricle space, hippocampus size, and hypo- and hyper-intensity area.

\section{RESULTS}

Evaluation of T2-weighted MRI: The volume of lateral ventricle space (mean: 1,700 $\mathrm{mm}^{3}$; range: $149-5,960 \mathrm{~mm}^{3}$ ) in the dogs was remarkably varied, however, it showed a significant positive correlation with the aging. No correlation was observed between the size of hippocampus and the aging (Figs. 1 and 2). The hypo-intensity area, decreasing of signal intensity on T2-weighted images, was detected in both globus pallidus and substantia nigra in aged beagle dogs from 7-year-old (Fig. 1). Both intensity ratios of globus pallidus and substantia nigra to lateral ventricle were significantly correlated with age advanced (Fig. 3). In addition, hyper-intensity area, showing mild increase of signal intensity, was detected in white matter surrounding lateral ventricle on T2-weighted image in 16-year-old beagle dogs (Fig. 4).

Pathological examination: The brain of the 16-year-old beagle dog showed atrophy and lateral ventricular dilatation on macroscopic observation. The ferric iron was clearly detected in the globus pallidus and substantia nigra as a blue area stained by the Perls' solution. The regions of the area were coincided with hypo-intensity area on T2-weighted MRI (Fig. 5).

\section{DISCUSSION}

In this study, the volume of the lateral ventricle space in the brain showed a significant correlation with the aging in beagle dogs. In aged human brain, the dilation of cerebrospinal fluid space was observed on MRI and also computed tomography, indicating atrophy of brain and also decrease number of neuronal cell [7, 25, 31]. Atrophy of hippocampus, which was closely related to the memory function, was also reported in human with age advanced

A
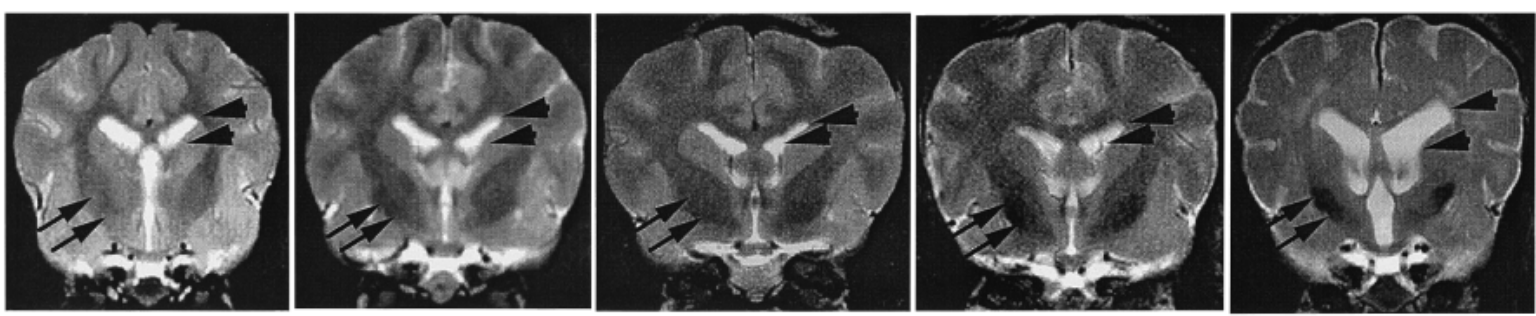

B

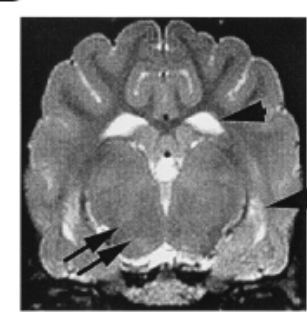

1-year-old

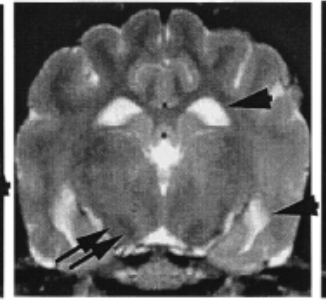

7-year-old

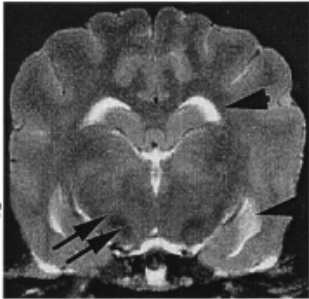

10-year-old

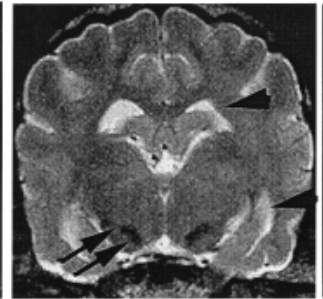

12-year-old

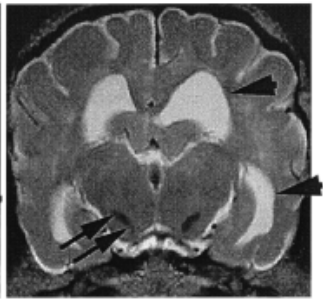

16-year-old

Fig. 1. Typical transverse sections of T2-weighted spin-echo images in magnetic resonance imaging (MRI) at the level of globus pallidus (A) and substantia nigra (B) in various aged beagle dogs. Arrowheads and arrows indicate lateral ventricle and hypo-intensity area, respectively. 

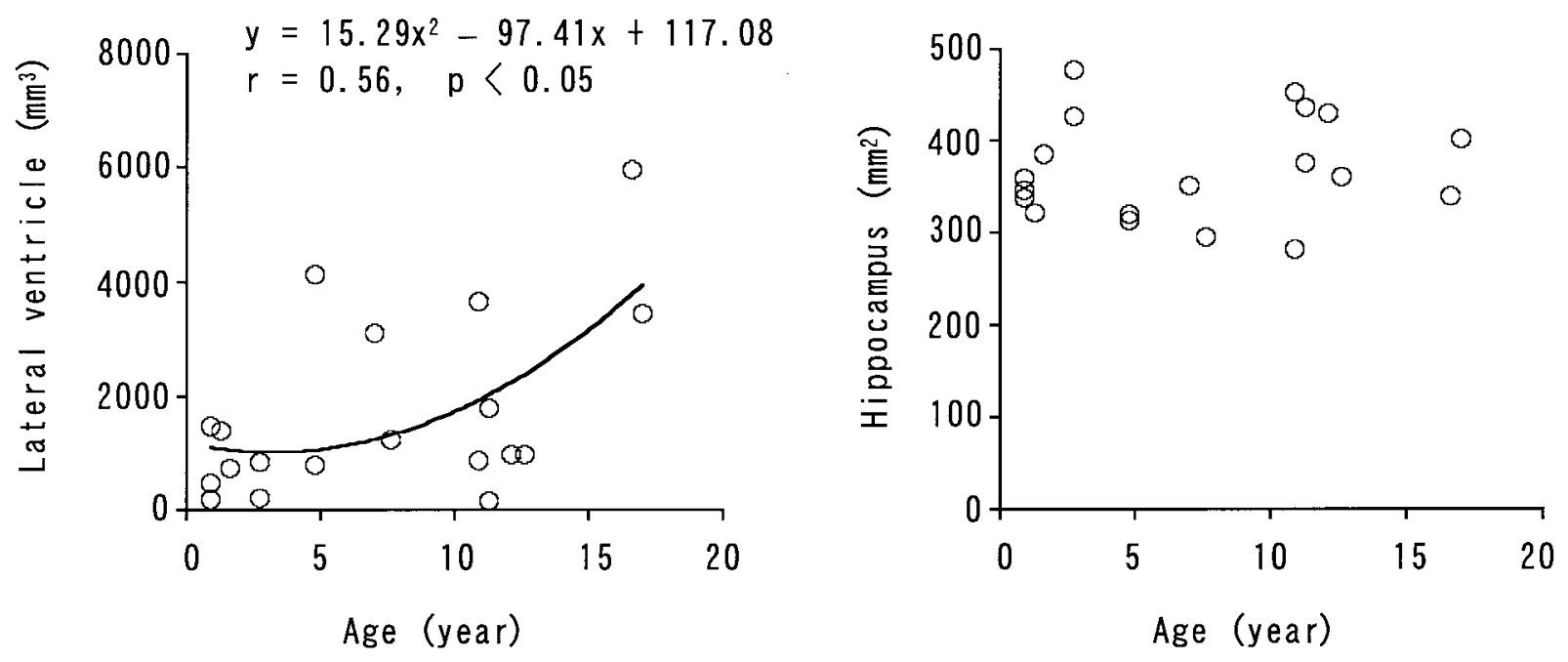

Fig. 2. Correlation between the volume of lateral ventricle space (A) or hippocampus size (B), and the aging in beagle dogs. A significant positive correlation was observed between the volume of lateral ventricle space and the aging.

A

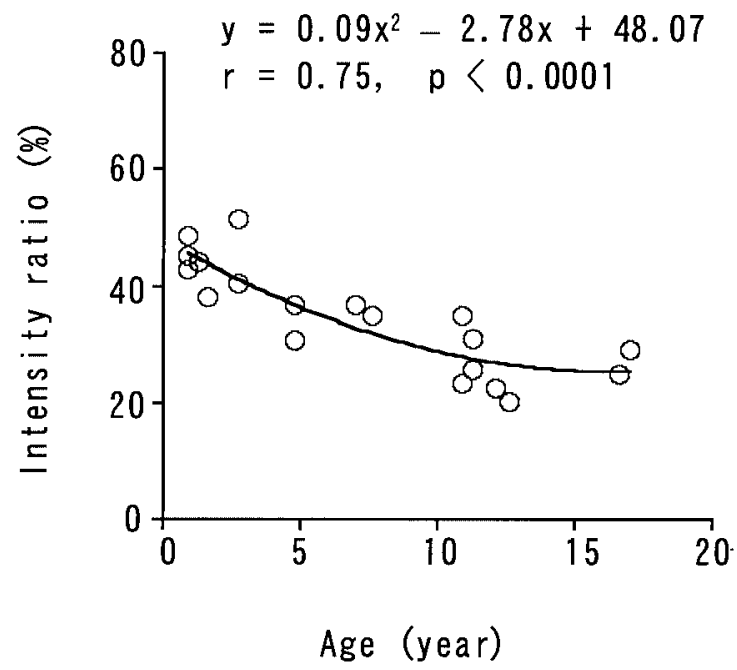

B

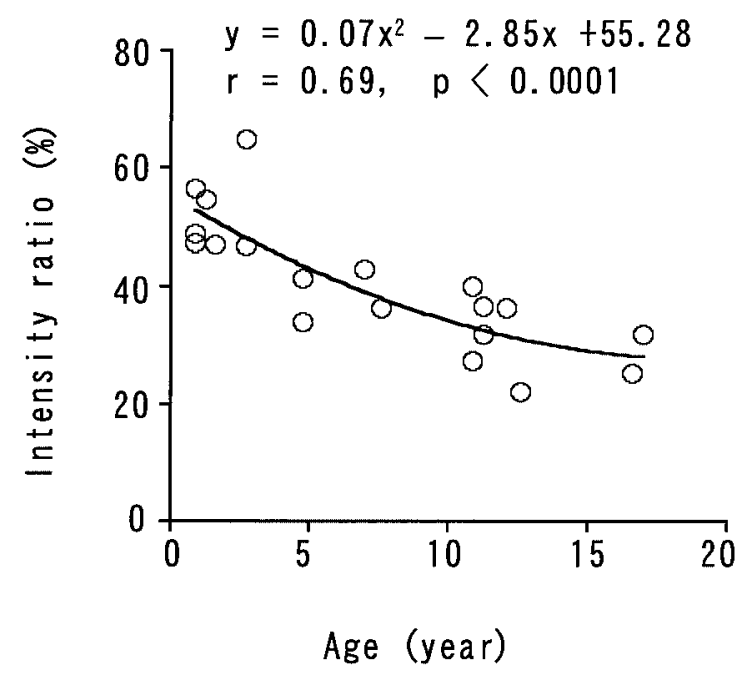

Fig. 3. Correlation between the intensity ratio of globus pallidus (A) or substantia nigra (B) to lateral ventricle, and the aging. A significant positive correlation was observed in both ratios.

$[11,20]$. In aged dogs, any atrophy of hippocampus could not detected, although it was the major age-associated finding in human. Age-associated changes might be different among animals, resulted in different manners and/or behaviors in dementia symptoms.

Hypo-intensity area on T2-weighted images was observed in both globus pallidus and substantia nigra in bea- gle dogs with age advanced. Aoki et al. [1] reported that hypo-intensity area on T2-weighted images was commonly observed in substantia nigra, red nucleus, and globus pallidus from age 25 years in the brain of clinically healthy human. Many researchers indicated that the main cause of this was non-haemin iron accumulation $[3,14]$. The highest accumulated region with iron was reported to be in globus 

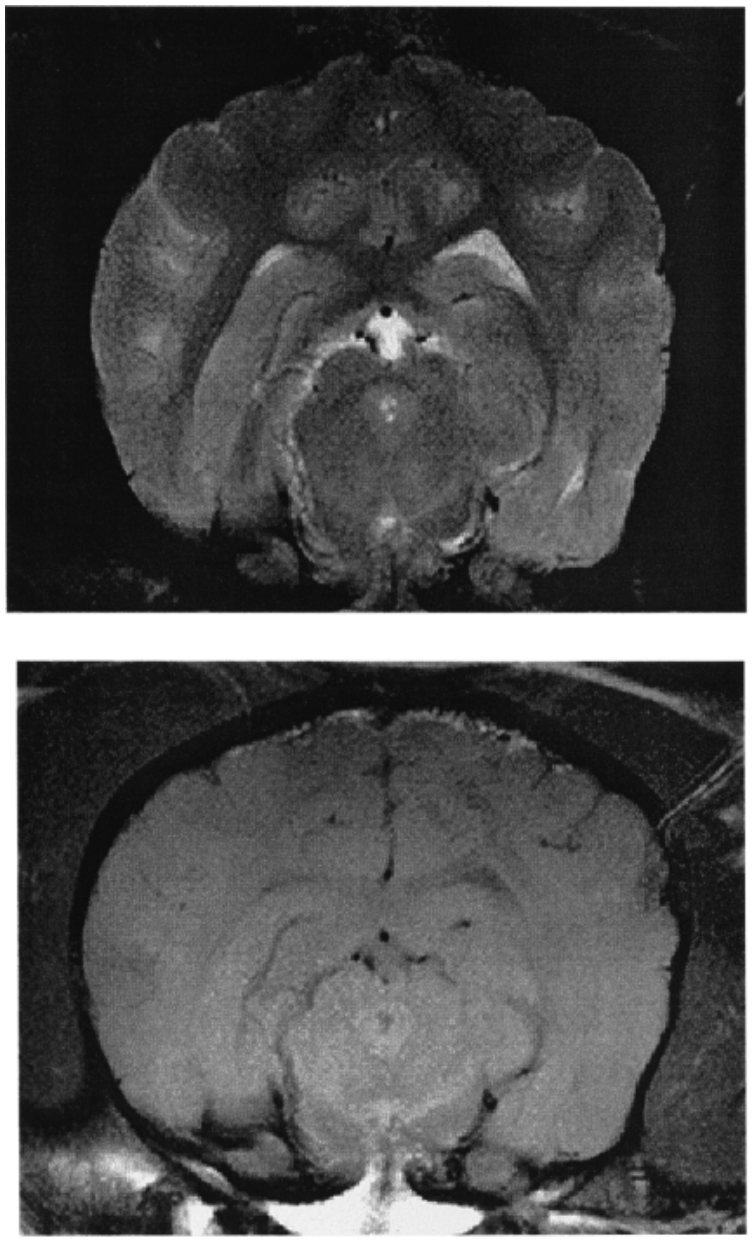

2-year-old
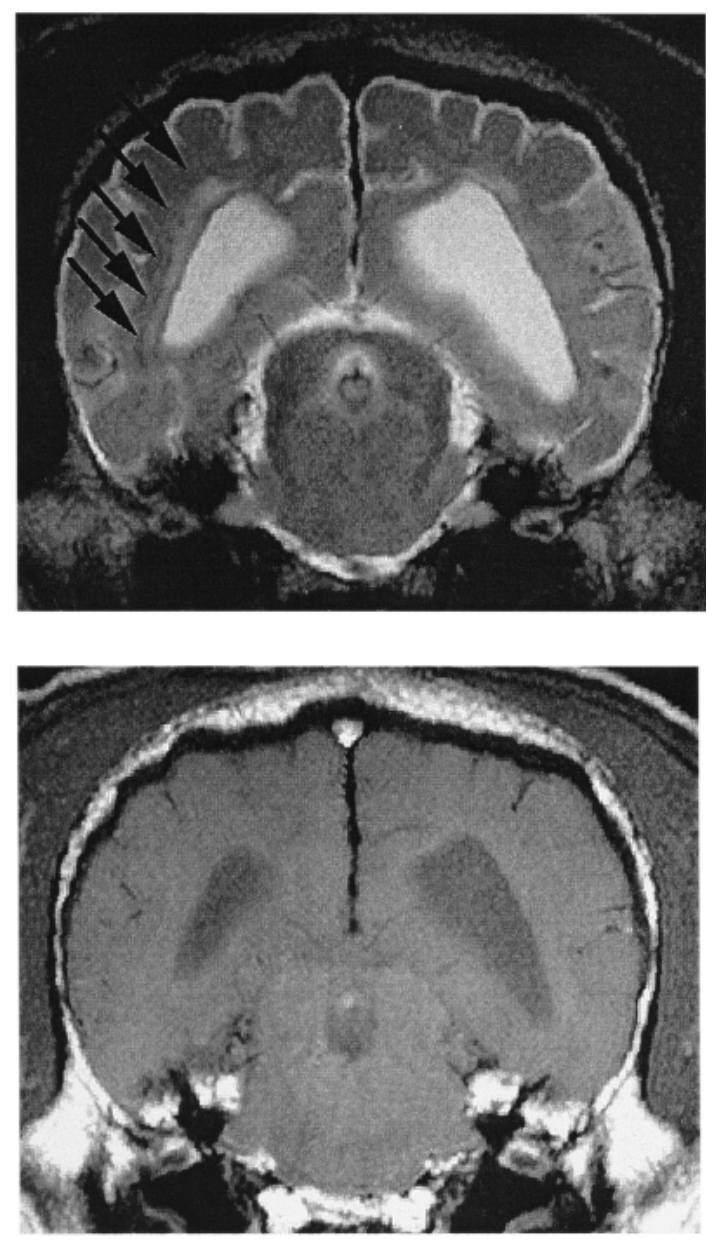

16-year-old

Fig. 4. Typical transverse sections of T2-weighted (upper) and T1-weighted (lower) spin-echo images in magnetic resonance imaging (MRI) at the level of midbrain of the 2-year-old (left) and 16-year-old (right) beagle dog. Hyper-intensity area on T2-weighted MRI was detected in white matter surrounding lateral ventricle in the 16-year-old dog (arrows).

pallidus, followed by red nucleus and substantia nigra in the human brain, like as reported on hypo-intensity area of T2weighted MRI [2, 22]. The hypo-intensity areas on T2weighted images were also detected in caudate nucleus, putamen, and globus pallidus in the monkey brain [3], and in globus pallidus and substantia nigra in the dog brain, both of which were closely related to iron deposition with the aging. The meaning of iron accumulation in the brain was poorly understood, however, it was considered to affect the metabolism of neurotransmitters and/or neuroactive compounds. Hill and Switzer [24] demonstrated the distribution of iron was overlapped with gamma-aminobutyric acid (GABA), enkephalin and lutenizing hormone releasing hormone (LHRH) in the rat brain. The increase of iron concentration in the brain was also detected in several neurological disorders, such as multiple sclerosis, Shy-drager syndrome, Parkinson's disease, and abnormal movement disorders [15, 21, $33,35]$. It was widely accepted that iron generated oxygen free radicals, resulted in neurological cell death $[32,36]$ and also disturbance of neuronal functions in human with age advanced [39]. Although the beagle dogs used in this study showed no abnormalities in their gait and behaviors, accumulation of iron in globus pallidus and substantia nigra with the aging was suggested to be some affects on the neuronal functions in aged dogs.

Hyper-intensity area was detected in white matter surrounding the lateral ventricle on T2-weighted images in the 16 -year-old beagle dog. The same lesion was often observed in the clinically healthy aged human brain. Gerard and Weisherg [19] reported that $7.8 \%$ of human over age 50 years showed perivascular hyper-intensity area on T2weighted images without symptoms of cerebrovascular disease. The area was resulted from the changes of myelin structure with fluid-filled space in white matter [19, 29]. Since the degeneration of white mater was reported in dogs aged more than 14-year-old [18], the hyper-intensity area on 

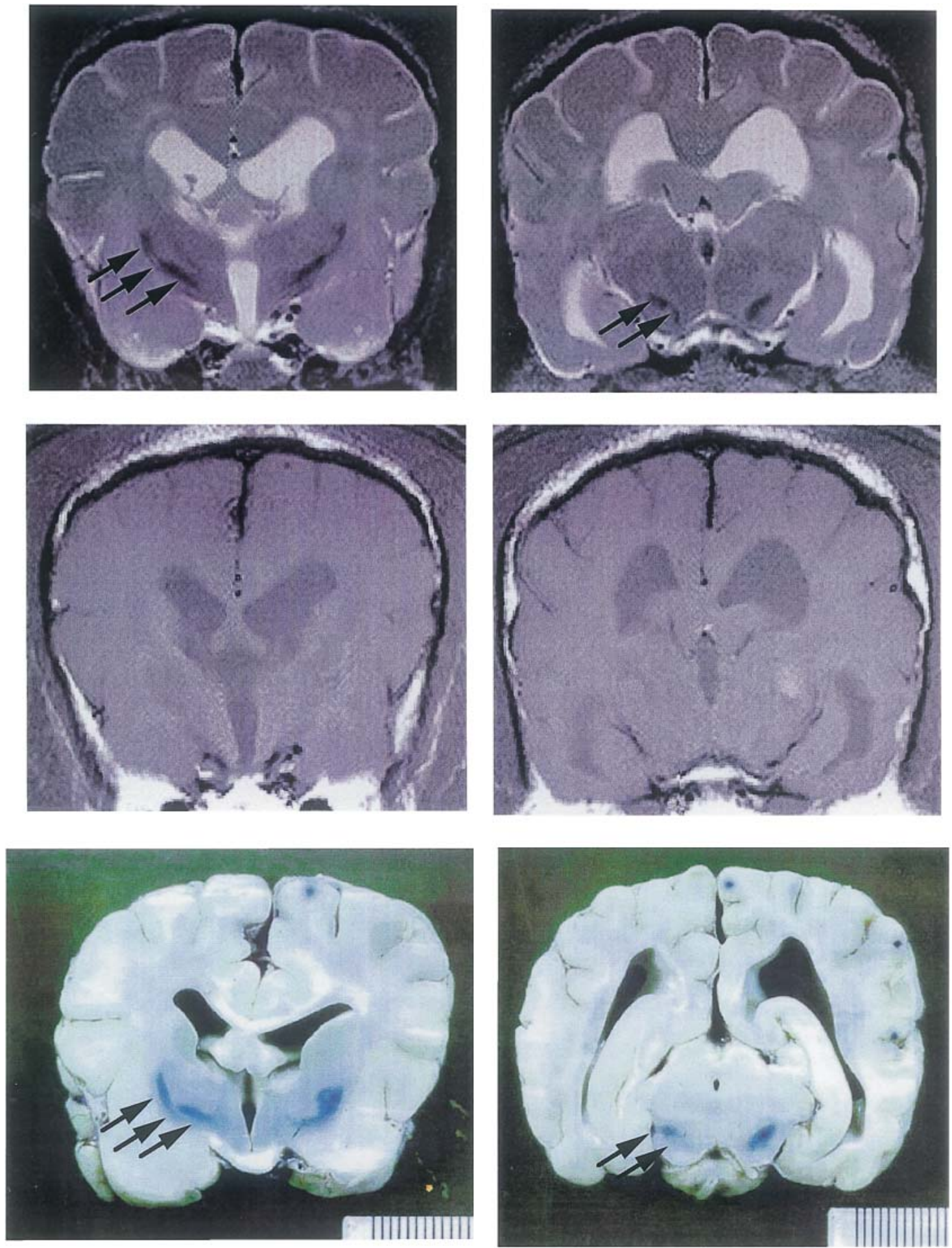

Fig. 5. Transverse sections of T2-weighted (upper) and T1-weighted (middle) spin-echo images in magnetic resonance imaging (MRI) and slice sections with Perls' staining (lower) at the level of globus pallidus (left) and substantia nigra (right) in the 16-year-old beagle dog. The hypo-intensity area on T2-weighted MRI was coincided with the region with accumulated iron as the blue area by Perls' staining (arrows). 
T2-weigted images was considered to be one of the ageassociated changes in white matter in the dog brain, although the area observed in beagle dogs was slightly mild compared with that in the human.

From these results, age-associated changes of T2weighted MRI were developed in the dog brain, especially in globus pallidus, substantia nigra, and white matter surrounding lateral ventricle, like as those reported in the human brain.

ACKNOWLEDGEMENT. This work was supported in part by a Grant-in-Aid for Scientific Research from Ministry of Education, Culture, Sports, Science and Technology of Japan (Nos. 14360189 and 16380211).

\section{REFERENCES}

1. Aoki, S., Okada, Y., Nishimura, K., Barkovich, A.J., Kjos, B.O., Brasch, R.C. and Norman, D. 1989. Normal deposition of brain iron in childhood and adolescence: MR imaging at 1.5 T. Radiology 172: 381-385.

2. Bartzokis, G., Mintz, J., Sultzer, D., Marx, P., Herzberg, J.S., Phelan, C.K. and Marder, S.R. 1994. In vivo MR evaluation of age-related increases in brain iron. Am. J. Neuroradiol. 15: 1129-1138.

3. Bizzi, A., Brooks, R.A., Brunetti, A., Hill, J.M., Alger, J.R., Miletich, R.S., Francavilla, T.L. and Di Chiro, G. 1990. Role of iron and ferritin in MR imaging of the brain: a study in primates at different field strengths. Radiology 177: 59-65.

4. Borras, D., Ferrer, I. and Pumarola, M. 1999. Age-related changes in the brain of the dog. Vet. Pathol. 36: 202-211.

5. Bush, W.W., Barr, C.S., Darrin, E.W., Shofer, F.S., Vite, C.H. and Steinberg, S.A. 2002. Results of cerebrospinal fluid analysis, neurologic examination findings, and age at the onset of seizures as predictors for results of magnetic resonance imaging of the brain in dogs examined because of seizures: 115 cases (1992-2000). J. Am. Vet. Med. Assoc. 220: 781-784.

6. Chapman, B.L. and Voith, V.L. 1990. Behavioral problems in old dogs: 26 cases (1984-1987). J. Am. Vet. Med. Assoc. 196: 944-946.

7. Coffey, C.E., Wilkinson, W.E., Parashos, I.A., Soady, S.A., Sullivan, R.J., Patterson, L.J., Figiel, G.S., Webb, M.C., Spritzer, C.E. and Djang, W.T. 1992. Quantitative cerebral anatomy of the aging human brain: a cross-sectional study using magnetic resonance imaging. Neurology 42: 527-536.

8. Coleman, P.D. and Flood, D.G. 1987. Neuron numbers and dendritic extent in normal aging and Alzheimer's disease. Neurobiol. Aging 8: 521-545.

9. Cuenod, C.A., Denys, A., Michot J.L., Jehenson P., Forette, F., Kaplan, D., Syrota, A. and Boller, F. 1993. Amygdala atrophy in Alzheimer's disease. An in vivo magnetic resonance imaging study. Arch. Neurol. 50: 941-945.

10. Cummings, B.J., Head, E., Ruehl, W., Milgram, N.W. and Cotman, C.W. 1996. The canine as an animal model of human aging and dementia. Neurobiol. Aging 17: 259-268.

11. De Leon, M.J., George, A.E., Golomb, J., Tarshish, C., Convit, A., Kluger, A., De Santi, S., McRae, T., Ferris, S.H., Reisberg, B., Ince, C., Rusinek, H., Bobinski, M., Quinn, B., Miller, D.C. and Wisniewski, H.M. 1997. Frequency of hippocampal formation atrophy in normal aging and Alzheimer's disease. Neurobiol. Aging 18: 1-11.
12. Dickson, D.W. 1997. The pathogenesis of senile plaques. $J$. Neuropathol. Exp. Neurol. 56: 321-339.

13. Drachman, D.A., Long, R.R. and Swearer, J.M. 1994. Neurological evaluation of the elderly patient. pp. 159-180. In: Clinical Neurology of Aging, 2nd ed. (Albert, M.L. and Knoefel, J.E. eds.), Oxford University Press, New York.

14. Drayer, B., Burger, P., Darwin, R., Riederer, S., Herfkens, R. and Johnson, G.A. 1986. MRI of brain iron. Am. J. Roentgenol. 147: 103-110.

15. Drayer, B., Burger, P., Hurwitz, B., Dawson, D. and Cain, J. 1987. Reduced signal intensity on MR images of thalamus and putamen in multiple sclerosis: increased iron content? Am. J. Roentgenol. 149: 357-363.

16. Drayer, B.P. 1988. Imaging of the aging brain. Part I. Normal findings. Radiology 166: 785-796.

17. Drayer, B.P. 1988. Imaging of the aging brain. Part II. Pathologic conditions. Radiology 166: 797-806.

18. Ferrer, I., Pumarola, M., Rivera, R., Zujar, M.J., Cruz-Sanchez, F. and Vidal, A. 1993. Primary central white matter degeneration in old dogs. Acta Neuropathol. 86: 172-175.

19. Gerard, G. and Weisberg, L.A. 1986. MRI periventricular lesions in adults. Neurology 36: 998-1001.

20. Golomb, J., de Leon, M.J., Kluger, A., George, A.E., Tarshish, C. and Ferris, S.H. 1993. Hippocampal atrophy in normal aging. An association with recent memory impairment. Arch. Neurol. 50: 967-973.

21. Gorell, J.M., Ordidge, R.J., Brown, G.G., Deniau, J.C., Buderer, N.M. and Helpern, J.A. 1995. Increased iron-related MRI contrast in the substantia nigra in Parkinson's disease. Neurology 45: 1138-1143.

22. Hallgren, B. and Sourander, P. 1958. The effect of age on the non-haemin iron in the human brain. J. Neurochem. 3: 41-51.

23. Hansen, L.A., Armstrong, D.M. and Terry, R.D. 1987. An immunohistochemical quantification of fibrous astrocytes in the aging human cerebral cortex. Neurobiol. Aging 8: 1-6.

24. Hill, J.M. and Switzer, R.C. 1984. The regional distribution and cellular localization of iron in the rat brain. Neuroscience 11: $595-603$.

25. Hughes, C.P. and Gado, M. 1981. Computed tomography and aging of the brain. Radiology 139: 391-396.

26. Murphy, D.G., DeCarli, C., Schapiro, M.B., Rapoport, S.I. and Horwitz, B. 1992. Age-related differences in volumes of subcortical nuclei, brain matter, and cerebrospinal fluid in healthy men as measured with magnetic resonance imaging. Arch. Neurol. 49: 839-845.

27. Kiatipattanasakul, W., Nakamura, S., Hossain, M.M., Nakayama, H., Uchino, T., Shumiya, S., Goto, N. and Doi, K. 1996. Apoptosis in the aged dog brain. Acta Neuropathol. 92: 242-248.

28. Killiany, R.J., Moss, M.B., Albert, M.S., Sandor, T., Tieman, J. and Jolesz, F. 1993. Temporal lobe regions on magnetic resonance imaging identify patients with early Alzheimer's disease. Arch. Neurol. 50: 949-954.

29. Kirkpatrick, J.B. and Hayman, L.A. 1987. White-matter lesions in MR imaging of clinically healthy brains of elderly subjects: possible pathologic basis. Radiology 162: 509-511.

30. Mosier, J.E. 1989. Effect of aging on body systems of the dog. Vet. Clin. North Am. Small Anim. Pract. 19: 1-12.

31. Murphy, D.G., DeCarli, C., Schapiro, M.B., Rapoport, S.I. and Horwitz, B. 1992. Age-related differences in volumes of subcortical nuclei, brain matter, and cerebrospinal fluid in healthy men as measured with magnetic resonance imaging. Arch. Neurol. 49: 839-845. 
32. Olanow, C.W. 1993. A radical hypothesis for neurodegeneration. Trends Neurosci. 16: 439-444.

33. Pastakia, B., Polinsky, R., Di Chiro, G., Simmons, J.T., Brown, R. and Wener, L. 1986. Multiple system atrophy (Shy-Drager syndrome): MR imaging. Radiology 159: 499-502.

34. Rusinek, H., de Leon, M.J., George, A.E., Stylopoulos, L.A., Chandra, R., Smith, G., Rand, T., Mourino, M. and Kowalski, H. 1991. Alzheimer disease: measuring loss of cerebral gray matter with MR imaging. Radiology 178: 109-114.

35. Rutledge, J.N., Hilal, S.K., Silver, A.J., Defendini, R. and Fahn, S. 1987. Study of movement disorders and brain iron by MR. Am. J. Roentgenol. 149: 365-379.

36. Ryan, T.P. and Aust, S.D. 1992. The role of iron in oxygenmediated toxicities. Crit. Rev. Toxicol. 22: 119-141.

37. Su, M.Y., Head, E., Brooks, W.M., Wang, Z., Muggenburg,
B.A., Adam, G.E., Sutherland, R., Cotman, C.W. and Nalcioglu, O. 1998. Magnetic resonance imaging of anatomic and vascular characteristics in a canine model of human aging. Neurobiol. Aging 19: 479-485.

38. West, C.D. 1979. A quantitative study of lipofuscin accumulation with age in normals and individuals with Down's syndrome, phenylketonuria, progeria and transneuronal atrophy. $J$. Comp. Neurol. 186: 109-116.

39. Zecca, L., Stroppolo, A., Gatti, A., Tampellini, D., Toscani, M., Gallorini, M., Giaveri, G., Arosio, P., Santambrogio, P., Fariello, R.G., Karatekin, E., Kleinman, M.H., Turro, N., Hornykiewicz, O. and Zucca, FA. 2004. The role of iron and copper molecules in the neuronal vulnerability of locus coeruleus and substantia nigra during aging. Proc. Natl. Acad. Sci. U.S.A. 101: 9843-9848. 